\title{
The Role of Argon Plasma Coagulation in Radiation Proctitis: Obtaining Real Clinical Data
}

\author{
Hasan Maulahela \\ Division of Gastroenterology, Department of Internal Medicine, Faculty of Medicine \\ Universitas Indonesia/Dr. Cipto Mangunkusumo General National Hospital, Jakarta
}

Radiation to the pelvis is one of the most important treatment in pelvic malignancy both as adjuvant or neoadjuvant. One of the most frequent complication of radiation treatment is proctitis. The incidence of radiation proctitis is related to dose of radiation, area of exposure, method of delivery and the use of medications.

Common presentation of radiation proctitis is rectal bleeding. Other symptoms such as diarrhea, urgency, rectal pain, or passage of mucus can be found. Radiation proctitis can be classified into acute proctitis which occurs immediately after initiation of radiotherapy and chronic proctitis which symptoms occur after months or years after cessation of therapy.1 Chronic radiation proctitis can have clinical manifestation such as bleeding, stricture, perforation, and bowel obstruction. The incidence of chronic radiation proctitis is estimated $2-20 \%{ }^{2}$

Management of chronic radiation proctitis include noninvasive and invasive methods. Noninvasive methods such as anti-inflammatory agents, sucralfate, hyperbaric oxygen therapy and the use of antioxidants. Invasive treatments are ablative procedures, endoscopic coagulation and surgery. ${ }^{3}$ There have been no large controlled trials evaluating the treatment of radiation proctitis, most of the experience come from small clinical trial or retrospective studies.

Endoscopic coagulation is one of treatment modality for radiation-induced bleeding. Coagulation method that has been used including YAG laser coagulation or argon plasma coagulation (APC). These coagulation methods based on delivery of thermal coagulation. Studies have shown that endoscopic coagulation give improvement of symptoms and decrease of recurrence. Based on current studies APC is the favored treatment for bleeding in chronic radiation proctitis.

APC is non-contact coagulation which uses high-frequency monopolar energy. APC has been demonstrated in the treatment of gastrointestinal vascular ectasia. APC has limited tissue penetration which is good for superficial mucosal bleeding with low risk of complications. Based on retrospective studies, patients who failed medical therapy APC helps to reduce symptoms and maintain hemoglobin level. ${ }^{4,5}$

Study by Siregar et al in Indonesia National Cancer Hospital show real data on APC efficacy in chronic radiation proctitis although with limited number of samples. This study shows $84 \%$ patient achieve clinical improvement. This result is similar with other study by Karamanolis et al which show $79 \%$ patient with mild and severe chronic radiation proctitis successfully treated with APC. 6,7

Currently there are no consensus on how many sessions of APC for chronic radiation proctitis. Studies show that on average two to three treatment session to achieve significantly better outcome. Improvements persisted for months after the treatments. Future studies should be aimed at the optimum setting, APC technique and number of sessions to achieve maximum clinical and endoscopic improvement.

\section{REFERENCES}

1. Denton A, Forbes A, Andreyev J, Maher EJ. Non surgical interventions for late radiation proctitis in patients who have received radical radiotherapy to the pelvis. Cochrane database Syst Rev 2002;1:CD003455.

2. Tagkalidis PP, Tjandra JJ. Chronic radiation proctitis. ANZ J Surg. 2001;71:230-7.

3. Do NL, Nagle D, Poylin VY. Radiation proctitis: current strategies in management. Gastroenterol Res Pract 2011;2011:917941.

4. Silva RA, Correia AJ, Dias LM, Viana HL, Viana RL. Argon plasma coagulation therapy for hemorrhagic radiation proctosigmoiditis. Gastrointest Endosc 1999;50:221-4.

5. Fantin AC, Binek J, Suter WR, Meyenberger C. Argon beam coagulation for treatment of symptomatic radiation-induced proctitis. Gastrointest Endosc 1999;49:515-8.

6. D’Amico G, Garcia-Tsao G, Pagliaro L. Karamanolis G, Triantafyllou K, Tsiamoulos Z, Polymeros D, Kalli T, Misailidis N, et al. Argon plasma coagulation has a longlasting therapeutic effect in patients with chronic radiation proctitis. Endoscopy 2009;41:529-31. 
7. Siregar L, Loho IM, Waspodo AS, Swadari R, Maharani BA. Argon plasma coagulation for the treatment of chronic radiation proctitis - real-world data from Indonesian National Cancer Center. Indones J Gastroenterol Hepatol Dig Endosc 2021;22:106-9. 